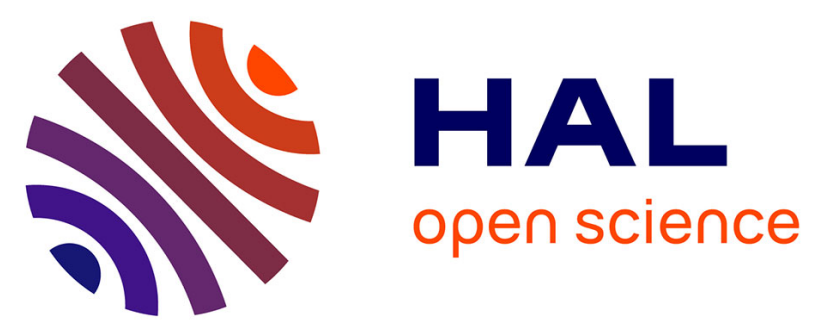

\title{
Numerical simulation of the thermodependant viscohyperelastic behavior of polyethylene terephthalate near the glass transition temperature: Prediction of the self-heating during biaxial tension test
}

Yun Mei Luo, Luc Chevalier, Françoise Utheza, E. Monteiro

\section{To cite this version:}

Yun Mei Luo, Luc Chevalier, Françoise Utheza, E. Monteiro. Numerical simulation of the thermodependant viscohyperelastic behavior of polyethylene terephthalate near the glass transition temperature: Prediction of the self-heating during biaxial tension test. Polymer Engineering and Science, 2013, on line 10.1002/pen.23522 (on line 10.1002/pen.23522), on line 10.1002/pen.23522. 10.1002/pen.23522 . hal-00833152

\section{HAL Id: hal-00833152 \\ https://hal.science/hal-00833152}

Submitted on 12 Jun 2013

HAL is a multi-disciplinary open access archive for the deposit and dissemination of scientific research documents, whether they are published or not. The documents may come from teaching and research institutions in France or abroad, or from public or private research centers.
L'archive ouverte pluridisciplinaire HAL, est destinée au dépôt et à la diffusion de documents scientifiques de niveau recherche, publiés ou non, émanant des établissements d'enseignement et de recherche français ou étrangers, des laboratoires publics ou privés. 


\section{Numerical Simulation of the Thermodependant Visco- hyperelastic Behaviour of PET near the Glass Transition Temperature: prediction of the self-heating during biaxial tension test}

Yun-Mei Luo ${ }^{* 1}$, Luc Chevalier ${ }^{1}$, Françoise Utheza ${ }^{1}$, Eric Monteiro ${ }^{2}$

1: Université Paris-Est, Laboratoire Modélisation et Simulation Multi Echelle, MSME UMR 8208 CNRS, 5 Bd Descartes, 77454 Marne-la-Vallée CEDEX 2, France 2: Laboratoire Procédés et Ingénierie en Mécanique et Matériaux (PIMM, UMR CNRS 8006), Arts et Métiers ParisTech, 151 boulevard de l'Hôpital, 75013 Paris, France 


\section{Abstract}

The polyethylene terephthalate near the glass transition temperature highlights a strongly non linear elastic and viscous behaviour when biaxially stretched at high strain rates representative of the injection stretch blow moulding process. A non linear visco-hyperelastic model, where characteristics are coupled to the temperature, has been identified from equi-biaxial tension experimental results. The weak form of this model is presented and implemented into a finite element code and validated by comparing numerical simulation of equi-biaxial testing with the analytical solution in the isothermal case. Considering the thermal aspects, an experimental study, where PET sheets are heated using infrared (IR for short) lamps is also presented. The modeling of the IR radiation of the sheet helps to identify the thermal properties of the PET. The thermal model is then implemented in the finite element code, coupled to the $2 \mathrm{D}$ visco-hyperelastic model. A discussion is made to justify the accuracy of the assumption made on homogeneity of the temperature field through the thickness. The simulation of the 2D plane stress equibiaxial test shows the important influence of the thermal aspects and the coupled thermo-mechanical software is used to quantify the self-heating phenomenon in the case of the biaxial elongations.

\section{Keywords}

Viscoelastic properties, thermal properties, simulations, nonlinear polymer 


\section{Numerical Simulation of the Thermodependant Visco- hyperelastic Behaviour of PET near the Glass Transition}

Temperature: prediction of the self-heating during biaxial

\section{tension test}

\section{Introduction}

The injection stretch blow moulding (ISBM) process which is managed at a temperature near or slightly above the glass transition temperature $T_{g}$ involves multiaxial large strains at high strain rate of the polyethylene terephthalate material (PET). The performance of a PET bottles produced by the ISBM process depends on many parameters. During the ISBM process, the PET behaviour exhibits a highly elasticity, a strain hardening effect and a strong viscous and temperature dependency. Therefore, many researches have been conducted on the rheological behaviour of PET: Marckmann et al. [1] proposed and used a hyperelastic modelling approach which could not take into account the strain rate effect. Gorlier et al. [2, 3] also used the hyperelastic type models, however, although these models have demonstrated some success in representing the PET behaviour at specific strain rates, they have been found to be unstable in the numerical simulation. Chevalier and Marco [4] have managed biaxial tension tests near $T_{g}$ with a range of strain rates from 0.02 to $2 \mathrm{~s}^{-1}$. They proposed a simple viscoplastic model [4] identified from these tension tests. This model has been used by Bordival et al. [5] in a numerical procedure based on simulations of the heating and blowing phases performed to optimise the stretch blow moulding process. Cosson et al. [6], then developed this viscoplastic model into an anisotropic version. The strain hardening effect observed during tension can be related with the strain induced modifications of the 
microstructure of PET but this viscoplastic model approach fails to represent the relaxation stage after tension which Chevalier and Marco [7] carried out from the relaxation tests. In their work, the relaxation time has been identified from these tests and clearly demonstrates the contribution of a viscous part in a highly elastic macromolecular network. This stress relaxation behaviour is the main characteristics associated with viscoelastic materials. Therefore, the viscoelastic model which take into account the strain hardening and strain rate effects have been used for ISBM process $[8,9]$. However, the classical viscoelastic models such as the Upper Convected Maxwell model [9] or the Giesekus model [10] do not adequately demonstrate the strain hardening effect. Buckley and Jones [11, 12] proposed a non linear viscoelastic model which is physically based on molecular network theory. Lately, Menary et al. [13] have examined three different constitutive models: hyperelastic model, creep model and a viscoelastic model (Buckley model) in the ISBM simulation using the finite element package ABAQUS/standard. They found that the Buckley model (viscoelastic model) gave a better result than the others two models in terms of predicting thickness distribution in the bottle. Inspired from Figiel and Buckley's work [14], Chevalier et al. $[15,16]$ have recently proposed a non linear incompressible visco-hyperelastic model to represent the complex constitutive behaviour of PET. Experimental uniaxial and biaxial tests performed on PET were carried out by Menary et al. [17] in Queen's University of Belfast. These tension tests were managed with various tension speeds (from $1 \mathrm{~s}^{-1}$ to $32 \mathrm{~s}^{-1}$ ), which are higher than in Chevalier and Marco's work [4]. The nonlinear forms of elastic and viscous characteristics were proposed. Here, we implement this complex visco-hyperelastic model into a finite element code in the matlab environment . The weak form of this 4 field model (velocity $\underline{V}$, the elastic left Cauchy Green tensor $\underline{\underline{B_{e}}}$ and the related pressure $p$ and $q$ for the incompressibility assumptions) is presented in the $2 \mathrm{D}$ specific plane stress case. It enables to reduce the number of field to 2 (velocity, the elastic left Cauchy Green tensor). Simulations of 
biaxial tests are managed in order to compare with the analytical solution in the isothermal conditions.

Effects of temperature, initial heating conditions or self-heating during the process, are of fundamental importance during the injection stretch blow moulding process of PET bottles. In the ISBM process, an initial preform is heated in an oven to the process temperature, which is near or slightly above $T_{g}$. Over this temperature $\mathrm{Tg}$, the mobility of the molecular chains in material PET affects the orientation and the microstructure (crystallization). The mechanical properties of PET are dependent on the microstructural morphology of the PET and strongly depend on the process temperature as well as on the strain rates. On the other hand, the low mobility of micromolecules and the high viscosity of the material generate dissipation of energy which leads to a self-heating phenomenon. This self-heating effect must be taken into account in the simulation. In contrast, many existing numerical studies of ISBM $[14,18]$ neglect heat transfer: the temperature has been assumed to be constant during the process and the deformation induced heat was neglected. These researches show that a numerical simulation neglecting the effect of temperature during stretch-blow moulding process could not accurately predict or model the orientation and crystallization, which are highly temperature dependent during the process. Therefore, it is essential to incorporate heat transfer to represent the mechanical properties of the final bottles. Schmidt et al. [19] developed their work [8] in ISBM simulation by developing a non-isothermal finite element simulation to embed heat transfer during the deformation process. However it did not show the significant improvement in terms of predicting thickness distribution and the force exerted by the stretch rod. Yang et al. [20, 21] continued the work of Menary et al. [13] in a 2D isothermal simulation to a $2 \mathrm{D}$ non-isothermal simulation by using the Buckley model to represent the PET behaviour. Significant nonlinear differentials have been found in temperature and strain in the bottle thickness. Here, we first identify the thermal properties 
from IR heating tests of PET sheets. The identified parameters are compared to classical values of the literature. Especially, the IR heating flux coming from IR lamps is studied using radiative laws adapted to the test geometry. A good correlation is discovered. Then, the thermal part coupled with the viscohyperelastic model for the mechanical part are used to perform the simulation. The simulation enables to quantify the self-heating during the biaxial tests.

In the first section of this work, we present the implementation of the proposed non linear incompressible visco-hyperelastic model into a finite element code developed with Matlab. We present a two-field finite element formulation: global velocity $\underline{V}$ and elastic Cauchy Green tensor $\underline{\underline{B_{e}}}$. Rectangular finite elements with quadratic and linear interpolations are employed for velocity and elastic left Cauchy Green tensor. A numerical simulation of 2D plane stress case is performed. It reproduce well the strain hardening effect.

In the second section, a procedure is proposed for the identification of the thermal parameters from experimental results of a test where PET sheets are heated using IR lamps. Sheets used in this study are made with the PET Arnite D00301 from DSM industries. The IR heating with IR camera is widely used in the experimental set-up for the heat transfer [22, 23]. The Monte Carlo method is used to identify the parameters from the temperature evolution measured on the front face (in regard of the lamps) and the rear face of the sheet. The heat capacity $C_{p}$ is considered as a function of the temperature while the other parameters (thermal conductivity, emmisivity, convection coefficient...) are assumed to be independent of the temperature. A large section is devoted to the comparison of the identified parameters with classical values of the literature, especially for the IR heating modelling. Moreover, the weak form describing thermal behaviour adapted to plane stress case is presented in the end of this section.. 
In the third section, in order to accurately simulate the ISBM process, the thermal and mechanical parts are put together. Non-linear mechanical and thermal equilibrium equations are solved with implicit schemes on the current deformed configuration, which is updated at each time step. Finally, the parameters identified in the proposed model have to be adjusted because the self-heating effect is not negligible and has an important effect on the viscous part of the model. Therefore, an optimization procedure is managed to adjust the characteristics of the PET for these visco-hyperelastic model expressions to represent conveniently the biaxial experimental tension tests [17].

\section{Numerical simulation of the model in plane stress case}

\subsection{Model presentation}

Inspired from Figiel and Buckley [14], we proposed in $[15,16]$ a non linear incompressible visco-hyperelastic model for both elastic and viscous parts to represent the mechanical behaviour. In order to represent the strain hardening and strain rate effect and temperature dependency, we choose two rheological functions for elastic and viscous parts: $G\left(\varepsilon_{e}\right)$ and $\eta\left(\varepsilon_{v}, \overline{\dot{\varepsilon}_{v}}, \mathrm{~T}\right)$

$$
\left\{\begin{array}{l}
\hat{\underline{\hat{\sigma}}}=2 G\left(\varepsilon_{e}\right) \hat{\hat{\varepsilon}}_{e} \\
\hat{\underline{\hat{\sigma}}}=2 \eta\left(\varepsilon_{v}, \overline{\underline{\overline{\dot{\varepsilon}_{v}}}}, T\right) \underline{\underline{D_{v}}}
\end{array} \quad \text { and } \quad \underline{\underline{\sigma}}=2 \eta_{N} \underline{\underline{D}}+\underline{\underline{\hat{\sigma}}}-p \underline{\underline{I}}-q \underline{\underline{I}}\right.
$$

where $\underline{\underline{\sigma}}$ is the Cauchy stress tensor, $\underline{D}_{v}$ is the symmetric part of the viscous velocity gradient, $\underline{\underline{D}}$ is the symmetric part of the global velocity gradient, $\underline{\underline{I}}$ is the identity matrix, $\varepsilon_{e}$ is the equivalent elastic strain, $\varepsilon_{v}$ is the equivalent viscous strain, $\overline{\dot{\varepsilon}_{v}}$ is the equivalent viscous strain rate, $T$ is the temperature, the subscript "^" denotes the deviatoric part of the tensor, $\eta_{N}$ is the small value of the viscosity of the Newtonian branch of the Zener like model used in order to 
solve the ill-conditioned problem, $\underline{\underline{\varepsilon_{e}}}$ is the elastic part of the Eulerian strain measure defined by:

$$
\underline{\underline{\varepsilon_{e}}}=\frac{1}{2}\left(\underline{\underline{B_{e}}}-\underline{\underline{I}}\right)
$$

where $\underline{\underline{B_{e}}}$ is the elastic left Cauchy Green tensor. $p$ is a Lagrange multiplier associated to the global incompressibility condition, and $q$ is the multiplier associated to the incompressibility of the elastic part. Since the elastic and global parts are incompressible, the viscous part is supposed to be also incompressible:

$$
\operatorname{det} \underline{\underline{B_{e}}}=1, \quad \operatorname{div} \vec{V}=\operatorname{trace} \underline{\underline{D}}=0, \quad \operatorname{div} \vec{V}_{v}=\underset{\underline{\underline{D_{v}}}}{\operatorname{trace}}=0
$$

where $\vec{V}$ is the global velocity and $\vec{V}_{v}$ is the viscous velocity.

The assumption of an additive decomposition of elastic and viscous velocity gradient is adopted to describe the kinematic structure of this model:

$$
\underline{\underline{D}}=\underline{D_{e}}+\underline{D_{v}}
$$

where $D_{e}$ is the symmetric part of the elastic velocity gradient.

Combining Eq. 1, 2 and 4 in the Oldroyd derivation of the elastic left Cauchy-Green tensor leads to:

$$
\frac{\delta \underline{\underline{B_{e}}}}{\delta t}+\frac{G}{\eta} \underline{\underline{B_{e}}} \underline{\underline{\hat{B}_{e}}}=0
$$

where $G$ is the elastic shear modulus, $\eta$ is the viscosity and the Oldroyd derivation $\delta \underline{\underline{B_{e}}} / \delta t$ is defined by:

$$
\frac{\delta \underline{\underline{B_{e}}}}{\delta t}=\underline{\underline{\dot{B}_{e}}}+\underline{\underline{B_{e}}} \underline{\underline{\Omega}}-\underline{\underline{\Omega}} \underline{\underline{B_{e}}}-a\left(\underline{\underline{B_{e}}} \underline{\underline{D}}+\underline{\underline{D}} \underline{\underline{B_{e}}}\right) \text { with } a=1 \text {. }
$$

where is $\underline{\underline{\Omega}}$ the global spin. 


\subsection{Identification of the material's properties}

Both the elastic and the viscous parts of the model must contribute to the strain rate effect. We first focus on the elastic part. One can identify the initial shear modulus $G_{0}$ from the initial slope of the global experimental strain-stress curves which were carried out by Menary et al. [17], because there is no viscous strain at the very beginning of the test. Table 1 show that $G_{0}$ does not vary much from one strain rate to another.

As the biaxial tests are conducted at constant nominal strain rate, the global strain rate decreases versus time. If the shear modulus $G$ remains constant, it leads to a contradiction because the viscous strain rate may become negative. Therefore, we consider a Hart-Smith like model to represent the elastic part:

$$
G\left(\varepsilon_{e}\right)=G_{0} \exp \left(\Lambda\left(I_{1}-3\right)^{2}\right), I_{1}=\operatorname{trace} \underline{\underline{\underline{B_{e}}}}
$$

where $\Lambda$ is a dimensionless parameter.

For the non-linear viscous part of the model we follow the same method as in Cosson et al. [6] to represent macroscopically the strain hardening effect, but we choose a Carreau type law instead of the power law to describe the influence of the strain rate:

$$
\begin{aligned}
\eta\left(\varepsilon_{v}, \overline{\dot{\varepsilon}_{v}}, T\right) & =\eta_{0} h\left(\overline{\varepsilon_{v}}\right) \cdot f\left(\overline{\dot{\varepsilon}_{v}}\right) \\
\text { with: } f\left(\overline{\dot{\varepsilon}_{v}}\right) & =\frac{1}{\left(1+\left(\lambda \frac{\overline{\dot{\varepsilon}_{v}}}{\dot{\varepsilon}_{r e f}}\right)^{a}\right)^{\frac{1-m}{a}}}
\end{aligned}
$$

where $\lambda, m, a$ are parameters in the Carreau type law and $\dot{\varepsilon}_{r e f}$ is a reference strain rate that can be taken equal to $1 \mathrm{~s}^{-1}$ for sake of simplicity. The strain hardening effect is related to the $h$ function which increases continuously with $\bar{\varepsilon}_{v}$. We detailed the identification procedure for the $h$ function in $[15,16]$, here we slightly changed the form of the function. Since the strain hardening effect is influenced by the temperature, $h$ is a function of $T$ too: 


$$
\eta_{0} h\left(\overline{\varepsilon_{v}}, T\right)=\frac{\eta_{0}(T) \cdot\left(1-\exp \left(-K \overline{\varepsilon_{v}}\right)\right)}{\left(1-\frac{\overline{\varepsilon_{v}}}{\varepsilon_{v \lim }(T)}\right)^{N}}
$$

According to the form of the lines of $h$ showed in the Fig. 1, we propose the function in the Eq. 9, where $\eta_{0}$ is related to the level of the function on the 'plateau', $K$ is a constant related to the initial slope of the curve, $\varepsilon_{v l i m}$ is the strain value corresponding to the vertical asymptote of the h curve and $N$ an exponent that fits the "beginning" of the quick increase of the curve.

Parameters $K$ and $N$ do not vary much with the temperature; at the contrary, variables $\eta_{0}(T)$ and $\varepsilon_{\text {vlim }}(T)$ show a significant dependence on temperature. We choose the Williams-LandelFerry (WLF) model for the evolution of $\eta_{0}(T)$ :

$$
\ln \left(a_{T}\right)=\frac{-C_{1}\left(T-T_{r e f}\right)}{C_{2}+T-T_{r e f}}, \eta_{0}(T)=a_{T} \eta_{0}\left(T_{r e f}\right)
$$

where $C_{1}$ and $C_{2}$ are the WLF parameters, $T_{r e f}=90^{\circ} \mathrm{C}$. We propose the evolution of $\varepsilon_{v l i m}(T)$ in the following way:

$$
\varepsilon_{v \lim }=\varepsilon_{v \lim _{\_} r e f}\left(1+\frac{B_{1}\left(T_{r e f}-T\right)}{\left(T-B_{2}\right)}\right)
$$

where $\varepsilon_{v \text { lim_ref }}=\varepsilon_{v \lim _{-} 90^{\circ} \mathrm{C}}$. Finally, the characteristics of the PET for these visco-hyperelastic model expressions to represent conveniently the experimental are listed in table 2.

\subsection{Weak form of the plane stress 2D visco-hyper-elastic problem}

In the equi-biaxial elongation plane stress case, before deriving the weak form, we can establish a relation between the pressures, the velocity and the elastic left Cauchy Green tensor:

$$
\sigma_{33}=0 \Rightarrow p+q=-2 \eta_{N}\left(D_{11}+D_{22}\right)+\frac{G\left(\varepsilon_{e}\right)}{3}\left(\frac{2}{B_{e 11} B_{e 22}-B_{e 12}^{2}}-B_{e 11}-B_{e 22}\right)
$$

The visco-hyperelastic model is implemented in the Matlab environment using a finite element approach. A 2 fields (global velocity $V$ and the elastic left Cauchy Green tensor $\underline{\underline{B_{e}}}$ ) 
variational formulation is proposed for plane stress incompressible problem. Some manipulations of Eq. 5, 6 and 12 lead to the following weak form:

$$
\begin{aligned}
& R_{V}=2 \eta_{N} \int_{\Omega} \underline{\underline{D}}^{*}: \underline{\underline{D}} d \Omega+G \int_{\Omega} \underline{\underline{D}}^{*} \underline{\underline{\hat{B}_{e}}} d \Omega+2 \eta_{N} \int_{\Omega} \underline{\underline{D}}^{*}: \underline{\underline{I}}\left(D_{11}+D_{22}\right) d \Omega \\
& -\frac{G}{3} \int_{\Omega} \underline{D}^{*}: \underline{I}=\frac{1}{B_{e 11} B_{e 22}-B_{e 12}{ }^{2}} d \Omega-\int_{\partial \Omega_{F}} \underline{V}^{*} \underline{F}^{d} d S=0 ; \\
& R_{B_{e}}=\int_{\Omega} \underline{\underline{B_{e}}} *:\left(\frac{\delta B_{e}}{\overline{\underline{B}}}+\frac{G\left(\varepsilon_{e}\right)}{\eta\left(\varepsilon_{v}, \overline{\dot{\varepsilon}_{v}}, T\right)} \underline{\underline{B_{e}}} \underline{\underline{\hat{B}_{e}}}\right) d \Omega=0
\end{aligned}
$$

where the superscript $*$ designates test quantities and $F^{d}$ the prescribed traction field over the boundary $\partial \Omega_{F}$ where the loads are imposed. The integral equations are studied on the entire volume $\Omega$.

This strongly nonlinear problem (finite elastic displacements, elastic left Cauchy Green tensor $\underline{\underline{B_{e}}}$, non constant shear modulus $G$ and viscosity $\left.\eta\right)$, is solved using a classical NewtonRaphson iterative procedure. The consistent linearization must be done with Gâteaux operators and the linear form of the problem for the increment $\Delta V$ and $\Delta B_{e}$ is written in the following system:

$$
\left[\begin{array}{ll}
{\left[D_{\Delta V}\left\{R_{V}\right\}\right]} & \left.\mid D_{\Delta B_{e}}\left\{R_{V}\right\}\right] \\
{\left[D_{\Delta V}\left\{R_{B_{e}}\right\}\right]} & {\left[D_{\Delta B_{e}}\left\{R_{B_{e}}\right\}\right.}
\end{array}\right]\left\{\begin{array}{l}
{[\Delta V]} \\
{\left[\Delta B_{e}\right]}
\end{array}\right\}=-\left\{\begin{array}{l}
{\left[R_{V}\right]} \\
{\left[R_{B_{e}}\right]}
\end{array}\right\}
$$

where $D_{\Delta V}\left\{R_{V}\right\}, D_{\Delta B_{e}}\left\{R_{V}\right\}, D_{\Delta V}\left\{R_{B_{e}}\right\}$ and $D_{\Delta B_{e}}\left\{R_{B_{e}}\right\}$ are the Gâteaux derivatives related to the increments:

$$
\begin{aligned}
D_{\Delta V}\left\{R_{V}\right\}= & \left.2 \eta_{n} \int_{\Omega} \underline{\underline{D}}^{*}: \underline{\underline{D(\Delta V)}} d \Omega+2 \eta_{n} \int_{\Omega} \underline{\underline{D}}^{*}:(\underline{\underline{(D(\Delta V)}}: \underline{\underline{I}}) \underline{\underline{I}}\right) d \Omega \\
D_{\Delta B_{e}}\left\{R_{V}\right\}= & \int_{\Omega} \underline{\underline{D}}^{*}:\left(G\left(\varepsilon_{e}\right) \underline{\underline{B_{e}}}\right) d \Omega-\int_{\Omega} \underline{\underline{D}}^{*}:\left(G\left(\varepsilon_{e}\right) \frac{1}{\Delta B_{e 11} \Delta B_{e 22}-\Delta B_{e 12}{ }^{2}} \underline{\underline{I}}\right) d \Omega \\
& \int_{\Omega} \underline{\underline{D}}^{*}:\left(D_{\Delta B}\left\{G\left(\varepsilon_{e}\right) \underline{\underline{B}}_{e}\right) d \Omega-\int_{\Omega} \underline{\underline{D}}^{*}:\left(\frac{1}{B_{e 11} B_{e 22}-B_{e 12}{ }^{2}} D_{\Delta B}\left\{G\left(\varepsilon_{e}\right)\right\} \underline{\underline{I}}\right) d \Omega\right.
\end{aligned}
$$




$$
\begin{aligned}
& \left.D_{\Delta V}\left\{R_{B_{e}}\right\}=2 \int_{\Omega} \underline{\underline{B_{e}}}: \underline{\underline{\underline{B}}} \underline{\underline{\Omega(\Delta V)}}\right) d \Omega-2 \int_{\Omega} \underline{\underline{B_{e}}}: \underline{\underline{\left(B_{e}\right.}} \underline{\underline{D(\Delta V)}} d \Omega \\
& \left.\left.D_{\Delta B_{e}}\left\{R_{B_{e}}\right\}=\int_{\Omega} \underline{\underline{B_{e}}}{ }^{*} \cdot \underline{\underline{\dot{B}_{e}}} d \Omega-2 \int_{\Omega} \underline{\underline{B_{e}}}{ }^{*}: \underline{\underline{\left(\Omega B_{e}\right.}}\right) d \Omega-2 \int_{\Omega} \underline{\underline{B_{e}}} *: \underline{\underline{D}} \underline{\underline{B_{e}}}\right) d \Omega \\
& +\int_{\Omega} \underline{\underline{B_{e}}} *\left(\frac{G\left(\varepsilon_{e}\right)}{\eta\left(\varepsilon_{v}, \overline{\dot{\varepsilon}_{v}}, T\right)} \underline{\underline{B_{e}}} \Delta \hat{B}_{e}\right) d \Omega+\int_{\Omega} \underline{\underline{B_{e}}} *:\left(\frac{G\left(\varepsilon_{e}\right)}{\eta\left(\varepsilon_{v}, \overline{\dot{\varepsilon}_{v}}, T\right)} \underline{\underline{\underline{B_{e}}}} \Delta B_{e}\right) d \Omega \\
& +\int_{\Omega} \underline{\underline{B_{e}}}:\left(D_{\Delta B}\left\{\frac{G\left(\varepsilon_{e}\right)}{\eta\left(\varepsilon_{v}, \overline{\dot{\varepsilon}_{v}}, T\right)}\right\} \underline{\underline{B_{e}}} \underline{\underline{\hat{B}_{e}}}\right) d \Omega
\end{aligned}
$$

Fig. 2 shows the geometry of the PET specimen during the tension tests, boundary conditions on symmetry axis and the load applied on the edge of the sheet. The PET sheet is submitted to a velocity $V$ on $\mathrm{X}$-axis and $\mathrm{Y}$-axis. This case represents the equal biaxial tension case: $\sigma_{x x}=\sigma_{y y}$. According to the axis of symmetry, the equal biaxial tension case can be simulated using one-quarter of the specimen. In order to compare the experimental results, the length and width of the $2 \mathrm{D}$ domain simulated are $38 \mathrm{~mm}$ and the thickness is $1.5 \mathrm{~mm}$, which is representative of the PET specimen size $(76 \mathrm{~mm} \times 76 \mathrm{~mm} \times 1.5 \mathrm{~mm})$ of the test [17].

As showed in the Fig. 2, the domain $\Omega$ is discretized by a set of 8 -nodes isoparametric rectangles elements. The simulation is managed for different elongation rate $\dot{\lambda}$ obtained from the derivative of stretch ratio $\lambda$ with respect to time $t$ : so $\dot{\varepsilon}=\dot{\lambda} / \lambda, \dot{\varepsilon}$ varies from $1 \mathrm{~s}^{-1}$ to $32 \mathrm{~s}^{-1}$.

\subsection{Simulation the biaxial plane stress testing}

In the case of the classical incompressible problem with a mixed velocity - pressure formulation the finite element calculations can lead to locking problem if velocity and pressure spaces are not chosen carefully. To be stable, a mixed formulation must verify consistency. The well-known inf-sup condition or the Ladyzenskaia-Babushka-Brezzi (LBB) condition [24] guaranties the stability of a finite element velocity - pressure calculation as a quadratic interpolation for velocity and linear for pressure. By analogy, we choose a quadratic interpolation for velocity $\underline{V}$ and linear interpolation for $\underline{\underline{B_{e}}}$. 
The finite elements result matches perfectly with the analytic results. Fig. $3 a$ and $3 b$ show a substantially good representation of the experimental results. The mean difference does not exceed $10 \%$.

\section{Numerical simulation of a thermo-mechanical model}

\subsection{Experimental procedure}

Mechanical properties of polymer are strongly influenced by small variation of temperature. On the other hand, the high viscosity of polymers generates important dissipation that impacts the temperature evolutions. Consequently, thermal properties are to be taken into account in the ISBM modeling in order to achieve accurate simulation of the process. It is necessary to:

(i) define accurately the initial temperature distribution of the preform at the beginning of the blowing operation;

(ii) identify the thermal properties of the PET in order to model the behaviour law of PET coupled to the thermal laws;

(iii) provide, by coupling the thermal equations with the mechanical equations, the history of the temperature field during the simulation.

In the following, a procedure is proposed for the identification of the thermal parameters. According to Fig. 4, the experimental apparatus which consists in measuring, by thermal imaging, a PET sheet heating by infrared lamps. A FLIR B250 infrared camera with the wavelength range $7.5-13 \mu \mathrm{m}$ is used to evaluate the surface temperature distribution. The surface dimension of the $1 \mathrm{~mm}$ thickness sheet is $60 \mathrm{~mm} \times 125 \mathrm{~mm}$. Sheets used in this study are injected from the PET Arnite D00301 following DSM industries recommendations.

We have tested three different distances between the lamps and the PET sheet: $11 \mathrm{~cm}, 12$ $\mathrm{cm}$ and $13 \mathrm{~cm}$. The thermal properties are identified for each distance: we can ensure the values of the heat conductivity, the specific masse, the heat capacity and the convective heat 
transfer parameters. Moreover, the relation between the heating flux and the distance can be estimated. For a constant heating IR flux, we can notice that the temperature decreases while this distance increases. This is a logical result because the intensity of the radiation decreases when the distance increases [25].

\subsection{Identification of the thermal properties}

The software FLIR quick report is used to measure accurately the temperature. In the work of Schmidt et al. [26], they have found that the polymer material is opaque under the wavelength in the range of $8-12 \mu \mathrm{m}$. This range matches the one of the IR camera so the PET may be considered like an opaque medium. The black paint used is assumed to be opaque which means that only the radiation emitted from the PET sheet surface is captured by the camera's sensor. To evaluate the temperature field from the IR camera, we need the emissivity value of the PET sheet. However, because we are not able to quantify it precisely, the identification is managed from thermocouple measures. From thermal imaging by camera, the temperature field visualization shows that some temperature heterogeneities only appear on the edges of the sheet surface. According to these results, we can assume that the temperature is homogeneous in the plane of the sheet and only varies in the thickness direction. Therefore, the identification can be done from a one dimensional model.

The heat transfer equation in the $1 \mathrm{D}$ case with the radiative source term can be written in the following way:

$$
\rho C_{p}(T) \dot{T}-k \frac{\partial^{2} T}{\partial^{2} z}=-\operatorname{div}\left(\overrightarrow{q_{r}}\right)
$$

where: $\rho$ the specific mass, $C_{p}$ the specific heat capacity, $k$ the material's conductivity and $\overrightarrow{q_{r}}$ is the internal radiative heat flux.

In Cosson et al. [27], the convective heat transfer coefficient for the face in front is little larger than the one on the rear face, but in other works [28], it is the opposite. Therefore, we 
choose to take into account the convection via two different coefficients. $T_{\infty}$ is the surrounding bulk temperature: $T_{\text {of }}$ for the air in front of the lamps is higher than the one in the back $T_{\infty r}$. Consequently, we write the boundary conditions in the following way:

$$
\begin{array}{ll}
-k \underline{\nabla T} \cdot \underline{n}=h_{f}\left(T-T_{\infty f}\right) \quad & \text { on the face in front of the lamps; } \\
-k \underline{\nabla T} \cdot \underline{n}=h_{r}\left(T-T_{\infty r}\right) \quad \text { on the rear face. }
\end{array}
$$

where $h_{f}$ and $h_{r}$ are the convective heat transfer coefficient on the face in front of the lamps and the one on the rear face.

The heat conductivity $k$, the specific mass $\rho$ and the convective heat transfer coefficient $h$ are assumed independent of temperature while the heat capacity $C_{p}$ is considered as a function of the temperature, since heat capacity increases significantly with the temperature (see Fig. 5). According to the values referenced in [29], we propose the following function to represent the evolution of the specific capacity:

$$
C_{P}=\Delta C_{p} \arctan \left(\alpha\left(T-T_{g}\right)\right)+C_{p 1}
$$

where $\Delta C_{p}$ is a constant related to the amplitude of the increased of the $C_{p}$ value when passing from the glassy state to the rubber state, $T_{g}$ is the more or less the glass transition temperature, $C_{p l}$ is a specific capacity value corresponding to the glassy state of the material and $\alpha$ is a factor that fits the roughness of the jump of the curve.

Since the heat transfer is assumed as a 1D case which the temperature varies only in the thickness direction, the internal radiative intensity absorption $\overrightarrow{q_{r}}$ is taken also as one dimensional and is managed by the Beer-Lambert law:

$$
\overrightarrow{q_{r}}=\phi_{\lambda 0} e^{-k_{\lambda} s} \vec{z}
$$


where: $\phi_{\lambda 0}$ is the incident radiation, $k_{\lambda}$ is the spectral absorption coefficient of PET and $s$ represent the path between the current position to the incident surface. From Fig. 8, s can be calculated as: $s=z-d$.

The heat equation (Eq. 16) with a non linear specific capacity (Eq. 18) and the equation of the internal heat flux (Eq. 19) are solved using a 1D finite element method. The implicit time integration scheme is chosen to solve this time-dependent problem. Due to the non linear specific capacity, a Newton Raphson method is used to obtain the temperature field.

The Monte Carlo method is used to identify the parameters that best fit the experimental results. The domain of each parameter is defined from the reference [29]. We generate the parameters randomly over the domain. On each draw, a computation is carried out and the numerical results are compared with the experimental temperatures. After drawing 10000 random inputs, we obtain the parameters, with which the numerical results best matches the experimental data. The thermal properties are referenced in table 3. This identification shows that $h_{f}$ is smaller than the rear coefficient $h_{r}$. The specific mass $\rho$ and the absorption coefficient $k_{\lambda}$ have the same order of magnitude with reference. The heat conductivity $k$ is smaller than the reference.

Fig. 6 represents for each distance, the experimental temperature evolution on the surface in front of the lamps $T_{f}$ (the blue one) and the one on the rear face $T_{r}$ (the red one). With the identified parameters, the curves obtained have a substantially good representation of the experimental results (dots).

The evolution of $C_{p}$ for PET Arnite D00301 are illustrated in Fig. 7, comparing with the one of material PET T4F9. They have the same order of magnitude except some differences at the lower temperature. The convective heat transfer coefficient $h$ can be estimated from the relation proposed by Churchill and Chu in [30]: 


$$
h=\frac{k}{L}\left(0.68+\frac{0.67 R_{a}^{1 / 4}}{\left(1+\left(0.492 / P_{r}\right)^{9 / 16}\right)^{4 / 9}}\right)
$$

where $L$ is the height of the sheet, $R_{a}$ is the Rayleigh number and $P_{r}$ is the Prandtl number. $P_{r}$ $=0.688$ and $R_{a}=1.6 \times 10^{6}$ when the temperature of PET reaches $100^{\circ} \mathrm{C}$ [29], we can obtain the value of $h$ using Eq. 20: $h=11 \mathrm{~W} / \mathrm{m}^{2} . \mathrm{K}$. The identified $h_{r}\left(16 \mathrm{~W} / \mathrm{m}^{2} . \mathrm{K}\right)$ is a little higher than this value estimated while $h_{f}\left(7 \mathrm{~W} / \mathrm{m}^{2} . \mathrm{K}\right)$ is a bit lower than this one.

Since we tested three different distances between the IR lamps and the PET sheet, the intensity of the incident radiation depends on the distance. In the following, we estimate this absorbed infrared radiation $\phi_{\lambda 0}$ from a simple modelling based on the principle of spectral energy relation [29,31]. Four identical IR lamps (radius $r=2 \mathrm{~mm}$ and length $l=185 \mathrm{~mm}$ ) are modelled as cylinders separated by a distance $d_{l}=15 \mathrm{~mm}$ (Fig. 8 b).

The amount of the radiation heat energy, that comes from the surface element $d A^{\prime}$ at a collocation point $M^{\prime}\left(x^{\prime}=r \cos \varphi+h, y^{\prime}, z^{\prime}=r \sin \varphi\right)$ and reaches the surface element $d A$ at a collocation point $M(x, y, z=d)$ with the path vector $\vec{w}$ (Fig.8a), can be written in the following way:

$$
d Q_{d A^{\prime} \rightarrow d A}=\int_{\lambda_{1}}^{\lambda_{2}} \varepsilon_{\lambda} i_{\lambda}^{b}(\lambda) d \lambda \cdot \int_{\varphi=0}^{\varphi=\pi} \int_{y^{\prime}=-l / 2}^{y^{\prime}=l / 2} \cos \theta \cos \theta^{\prime} \frac{d A}{\left\|M^{\prime} M\right\|^{2}} \underbrace{d A^{\prime}}_{r d \varphi d y^{\prime}}
$$

where $\lambda$ is a given wavelength between 0.2 and $10 \mu \mathrm{m}$ and $\varepsilon_{\lambda}$ is the spectral tungsten emissivity equal to 0.26 [29]. The emissive power for a blackbody $i_{\lambda}^{b}$ is given by Planck's law:

$$
i_{\lambda}^{b}=\frac{2 C_{1}}{\lambda^{5}\left(e^{C_{2} / \lambda T_{f i l}}-1\right)}
$$


where: $C_{1} \approx 1.19 \cdot 10^{8} \mathrm{~W} \cdot \mathrm{m}^{-2} \cdot \mu \mathrm{m}^{4}, C_{2} \approx 14388 \mu \mathrm{m} . \mathrm{K}$. We assume that the filament temperature is a uniform source at $T_{f i l}=1700 \mathrm{~K} . \vec{w}$ is a vector which represents the path of the radiation from $M^{\prime}$ to $M$ :

$$
\vec{w}=\frac{\overrightarrow{M^{\prime} M}}{\left\|M^{\prime} M\right\|}=\frac{x-x^{\prime}}{\left\|M^{\prime} M\right\|} \overrightarrow{e_{x}}+\frac{y-y^{\prime}}{\left\|M^{\prime} M\right\|} \overrightarrow{e_{y}}+\frac{z-z^{\prime}}{\left\|M^{\prime} M\right\|} \overrightarrow{e_{z}} \approx \frac{x-h}{\left\|M^{\prime} M\right\|} \overrightarrow{e_{x}}+\frac{y-y^{\prime}}{\left\|M^{\prime} M\right\|} \overrightarrow{e_{y}}+\frac{d}{\left\|M^{\prime} M\right\|} \overrightarrow{e_{z}}
$$

The last approximation is related to the condition $r \ll d$. The two angles $\theta^{\prime}$ and $\theta$ represent respectively, the angle between the normal at the lamp surface $\overrightarrow{n^{\prime}}$ at point $\mathrm{M}^{\prime}$ and the path direction $\vec{w}$; the angle between the normal at the PET sheet $\vec{n}$ at point M and the path direction $\vec{w}$ :

$$
\cos \theta=\vec{w} \cdot \vec{e}_{z} \approx \frac{d}{\left\|M^{\prime} M\right\|}, \cos \theta^{\prime}=\vec{w} \cdot \vec{e}_{r}=\frac{(x-h) \cos \varphi}{\left\|M^{\prime} M\right\|}+\frac{d \sin \varphi}{\left\|M^{\prime} M\right\|}
$$

where: $\left\|M^{\prime} M\right\|=\sqrt{(x-h)^{2}+\left(y-y^{\prime}\right)^{2}+d^{2}}$.

Once again, because the radius $\mathrm{r}$ is negligible compared to the distance d, Eq. 21 combining with Eq. 24 leads to:

$$
d Q_{d A^{\prime} \rightarrow d A}=\int_{\lambda_{1}}^{\lambda_{2}} \varepsilon_{\lambda} i_{\lambda}^{b}(\lambda) d \lambda \cdot 2 r d^{2} \int_{y^{\prime}=-l / 2}^{y^{\prime}=l / 2} \frac{d A d y^{\prime}}{\left\|M^{\prime} M\right\|^{2}}
$$

Finally, the intensity per unit area of the incident radiation can be written as follow:

$$
\phi_{\lambda 0}(M)=\frac{d Q_{\text {lamps } \rightarrow d A}}{d A}=\int_{\lambda_{1}}^{\lambda_{2}} \varepsilon_{\lambda} i_{\lambda}^{b}(\lambda) d \lambda \cdot 2 r d^{2} \sum_{i=1}^{n} \int_{y^{\prime}=-l / 2}^{y^{\prime}=l / 2} \frac{d y^{\prime}}{\left\|M^{\prime} M\right\|^{2}}
$$

where $n=4$ stands for 4 lamps.

We can see, from Fig. 9, that the intensity of the incident radiation reaching the PET sheet is not uniform. The maximum difference can reach $21 \%$ between the central zone of the PET sheet and the corners. Nevertheless, in the central region of study where the identification is 
done, the heterogeneity is less than $10 \%$ which validates the $1 \mathrm{D}$ approach used to manage the identification.

From Fig. 9d, one can also notice that the incident radiation $\phi_{\lambda 0}$ calculated in the central zone decreases with increasing distance. This value is close to the one identified from temperature measurements: both are listed in table 4.

\subsection{Implementation of the heat part of the thermo-visco-hyper-elastic model}

We consider a thermo-mechanical model to simulate the equibiaxial stretching of PET sheets in order to evaluate the self heating phenomenon. The mechanical part is shown in Eq. 13-15. The weak form of the heat part can be written in the following way:

$$
\left\{\begin{array}{l}
\int_{\Omega} T^{*} \rho C_{p} \dot{T} d \Omega+k \int_{\Omega} \underline{\nabla} T^{*} \underline{\nabla} T d \Omega=\int_{\Omega} T^{*}(\underline{\underline{\sigma}}: \underline{\underline{D}}) d \Omega-h \int_{\partial \Omega_{q}} T^{*}\left(T-T_{\infty}\right) d S \\
T=T_{0} \quad \text { at } \quad t=0
\end{array}\right.
$$

$\partial \Omega_{q}$ is the union of the top and bottom face of the specimen. The process temperature is slightly above the temperature Tg. Under this condition, based on the evolution of the heat specific capacity $C_{p}$ shown in Fig. 7 , it can be assumed as constant (1750 J/kg.K). Because the sheet specimen is heated on both sides, the heat transfer coefficient $h$ can be chosen equal to $h_{f}\left(7 \mathrm{~W} / \mathrm{m}^{2} \cdot \mathrm{K}\right)$. No thermal exchange is assumed between the specimen and the grips. To be consistent with the plane stress assumption, $T$ is chosen as a function of the plane coordinates $x, y$ and time (i.e., $T$ is representative of the mean value of the temperature through the thickness $e$ ). Consequently, the weak form writes:

$$
e\left(\rho C_{p} \int_{\partial \Omega} T^{*} \frac{\partial T}{\partial t} d S+k \int_{\partial \Omega} \underline{\nabla} T^{*} \underline{\nabla} T d S-\int_{\partial \Omega} T^{*}(\underline{\underline{\sigma}}: \underline{\underline{D}}) d S\right)=2 \int_{\partial \Omega_{q}} T^{*}\left(-h\left(T-T_{\infty}\right)\right) d S
$$

where $e$ is the thickness of the specimen. $\partial \Omega=\partial \Omega_{\mathrm{q}}=\mathrm{S}$ is the area of the $2 \mathrm{D}$ plane domain that represents the specimen. Since we assume that the thermal exchange only occurs on the 
top and bottom face of the PET sheet, the factor '2' before convective heat transfer term represents the total flux exchange from these two surfaces.

The dimension of the PET specimen during the test is $76 \mathrm{~mm} \times 76 \mathrm{~mm} \times 1.5 \mathrm{~mm}$. The length and width are large with respect to thickness: e $<$ L. Under this condition, the most convective heat exchange is on the top and bottom surfaces. The dissipated power density $\underline{\underline{\sigma}}: \underline{\underline{D}}$ is about $10^{6} \mathrm{~Pa} \cdot \mathrm{s}^{-1}$ and is almost uniform in the specimen. This value leads to the Brinkman number $B_{r}$ equals around 10:

$$
B_{r}=\frac{\eta \bar{V}^{2}}{k\left(T_{P}-\bar{T}\right)} \frac{e^{2}}{L^{2}}
$$

That means the viscous dissipation is 10 times larger than the heat conduction through the thickness.

Furthermore, the biaxial stretching process is considered fast enough in regard of the time needed to propagate the temperature through the thickness. The characteristic time for diffusion is $t_{d}=\rho C_{p} e^{2} / k=57 \mathrm{~s}$ and the characteristic time for capacity is $t_{c}=\rho C_{p} e / h=132 \mathrm{~s}$. The time for the process is about $2 \mathrm{~s}$. If we compare the mean value of the temperature through the thickness $\bar{T}=\bar{T}(x, y, t)$ in the plane stress case with the $T(x, y, z, t)$ in the axi-symmetric case, the difference between the mean value of the temperature $\bar{T}$ obtained by Eq. 28 and the temperature $\mathrm{T}$ calculated from the weak form with the definite integral over the thickness is nearly $0.55^{\circ} \mathrm{C}$. This difference causes an error on viscosity that is less than $3 \%$. Therefore, in the following, we use Eq. 28 to simulate the thermal part.

\subsection{Optimization of the mechanical parameters of the thermo-visco- hyper-elastic model}

Mechanical and thermal balance equations are fully non-linear and solved together with implicit schemes on the current deformed configuration, which is updated at each time step. The thermal parameters are identified from the experimental infrared heating and the 
mechanical ones are identified from the assuming isothermal equi-biaxial elongation. Both are listed in the table 2 and 3.

We implement the thermal part in the finite element approach together with the mechanical part. Therefore, a three fields (global velocity $V$, the elastic left Cauchy Green tensor $B$ and the temperature $T$ ) formulation has been performed. We choose the linear interpolation for temperature. The Eq. 13-15 which solve only the mechanical part has to also add the heat equations. The Newton-Raphson residual for the heat part is:

$$
\left.R_{T}=\rho C_{p} \int_{\partial \Omega} T^{*} \frac{\partial T}{\partial t} d S+k \int_{\partial \Omega} \underline{\nabla} T^{*} \underline{\nabla} T d S-\int_{\partial \Omega} T^{*} \underline{\underline{(\sigma}}: \underline{\underline{D}}\right) d S-2 / e \int_{\partial \Omega_{q}} T^{*}\left(-h\left(T-T_{\infty}\right)\right) d S
$$

The consistent linearization leads to the linear form of this problem for the increment $\Delta V$, $\Delta B_{e}$ and $\Delta T$ which can be given in the following way:

$$
\left[\begin{array}{ccc}
{\left[D_{\Delta V}\left\{R_{V}\right\}\right]} & {\left[D_{\Delta V}\left\{R_{V}\right\}\right]} & {[0]} \\
{\left[D_{\Delta V}\left\{R_{B}\right\}\right]} & {\left[D_{\Delta B}\left\{R_{B}\right\}\right]} & {\left[D_{\Delta T}\left\{R_{B}\right\}\right]} \\
{\left[D_{\Delta V}\left\{R_{T}\right\}\right]} & {[0]} & {\left[D_{\Delta T}\left\{R_{T}\right\}\right]}
\end{array}\right]\left\{\begin{array}{l}
{[\Delta V]} \\
{[\Delta B]} \\
{[\Delta T]}
\end{array}\right\}=-\left\{\begin{array}{l}
{\left[R_{V}\right]} \\
\left.\left[R_{B}\right]\right\} \\
{\left[R_{T}\right]}
\end{array}\right\}
$$

The adding Gateaux derivatives of nonlinear operator corresponding to the residuals $\left(R_{V}\right.$, $R_{B}$ and $R_{T}$ ) can be written:

$$
\begin{aligned}
& D_{\Delta T}\left\{R_{B}\right\}=\int_{\Omega} \underline{\underline{B_{e}}}: G\left(\underline{\left.\underline{\varepsilon_{e}}\right)} \underline{\underline{B_{e}}} \underline{\underline{\hat{B}_{e}}} D_{\Delta T}\left\{\frac{1}{\eta\left(\varepsilon_{v}, \dot{\bar{\varepsilon}}_{v}, T\right.}\right)\right\} d \Omega \\
& D_{\Delta V}\left\{R_{T}\right\}=-\int_{\Omega} T^{*}(\underline{\underline{\sigma}}: \Delta \underline{\underline{D}}) d \Omega \\
& D_{\Delta T}\left\{R_{T}\right\}=\int_{\Omega} T^{*} \rho C_{p} \Delta \dot{T} d \Omega+k \int_{\Omega} \underline{\nabla} T^{*} \underline{\nabla}(\Delta T) d \Omega
\end{aligned}
$$

Fig. 10 shows that stresses obtained from this thermo-mechanical simulation are lower than the experimental data. Because the temperature increases during the biaxial elongation, the self-heating effect affects the mechanical properties, especially the viscosity which decreases. Consequently, the parameters identified assuming an isothermal elongation must be modified to take into account this. 
As the self-heating effect is not negligible and produces an important effect on the viscous part of the model, the first estimation of the parameters obtained from our isothermal identification must be adjusted.

The parameters in the viscosity $\eta\left(\varepsilon_{v}, \overline{\dot{\varepsilon}_{v}}, T\right)$ are needed to optimize. The purpose is to minimise the mean difference between the experimental results and the numerical ones. The function 'fminunc' in the Matlab Optimization Toolbox is then chosen for the optimisation procedure. The characteristics identified from the isothermal elongation in table 2 are applied as the starting point of each parameter. Finally, the characteristics of the PET for these viscohyperelastic model expressions to represent conveniently the biaxial experimental tension tests are listed in the table 5.

Comparing with the values of table 2 , one can observe that only the values of the WLF like coefficients are influenced by this adjustment. Other coefficients, that appear in the mechanical part of the model vary very few: the initial identification makes finally sense even if an adjustment is needed a posterior.

\section{Results}

With these parameters identified from the optimization, we can obtain the stress-strain curves from the thermo-viscohyperelastic model. Fig. 11 shows the comparison between the stresses obtained from this thermo-mechanical simulation and from the experimental data. The mean difference does not exceed $10 \%$ for each deformation rate showed in table 6 .

We can notice that the increasing evolution of the temperature versus strain is nearly linear. The increase of the temperature is the same order of magnitude but little lower than the one observed in the experimental test presented in [17]. Furthermore, the self-heating of the specimen increases regularly for strain rates varying from $1 \mathrm{~s}^{-1}$ to $32 \mathrm{~s}^{-1}$ where the temperature increases nearly of $6^{\circ} \mathrm{C}$ (see Fig. $11 \mathrm{~b}$ ). It confirms that at very high deformation rate observed 
during ISBM process, the adiabatic heating due to the viscous dissipation may generate a significant temperature rises.

We can see in the Fig. 11c, the effect of temperature on the viscosity evolution. For example, the final slope of the viscosity curve for the strain rate $32 \mathrm{~s}^{-1}$ is much lower than the one for lower strain rates, therefore the strain hardening effect is not so considerable at $32 \mathrm{~s}^{-1}$.

If we examine the high strain rate case, for example, at $32 \mathrm{~s}^{-1}$ for $90^{\circ} \mathrm{C}$, the coupled themovisco-hyperelastique model simulation gives the stress evolution plotted on Fig. 11a which have $9.25 \%$ errors with the experimental data.

One can see that the stress evolution measured during the experiment saturates and doesn't increase as the lower strain rate case when approaching the 1.8 elongation. This may be explained by the higher temperature level coming from the self-heating of the specimen for this test. Our model does not predict the decreasing shape but gives a pretty good estimation on the evolution with nearly no strain hardening effect for this case.

\section{Conclusions}

A visco-hyperelastic model identified from the equi-biaxial tests performed at conditions close to ISBM process strain rate and temperature was implemented for numerical simulations. This finite elements model was used to simulate the plane stress test. It reproduces successfully the experimental results and can be used to simulate uniaxial or sequential biaxial tests to predict the PET behaviour for isothermal conditions.

Experiments have been conducted to characterise the thermal properties of the PET in the range of the ISBM temperature. Thermal imaging has been used to determine the surface temperature distribution of the PET sheets which are heated by infrared lamps. The Monte Carlo method is used to provide the parameters best fit the temperature evolution. Comparison between the obtained values and values coming from the literature, especially for the infrared heating radiation flux, validates the identification approach. 
The coupled thermo-visco-hyperelastic model proposed has been used to manage a finite element simulation of the equi biaxial elongation test. The weak form of the model has been implemented in Matlab. It shows that the thermal effects have an important influence on the viscous part of the model and WLF like parameters have to be adjusted by non-isothermal simulations. With the adjusted parameters, we obtained: (i) that stress-strain curves from this thermo-visco-hyperelastic model fit well with the experimental data; (ii) that self-heating of the specimen is not negligible and can reach nearly $10^{\circ} \mathrm{C}$ for the highest strain rate which is conform (slightly lower) to the temperature measurements made on the specimen.

In further works, we intend to implement an axi-symmetric version of the viscohyperelastic model coupled to temperature in order to simulate accurately the ISBM process.

\section{Acknowledgement}

This work could not possible without IR lamps given by Sidel Company and without injection of the PET sheets made by the LIM at Arts et Métiers ParisTech. Special thanks to G. Menary of QUB for his experimental study from which the identification has been possible.

\section{References}

[1] G. Marckmann, E. Verron, B. Peseux, Polymer Eng Sci., 41(3), 426-439 (2001).

[2] E. Gorlier, J.F. Agassant, J.M. Haudin, N. Billon, Plast Rubber Compos Process Appl., 30(2), 48-55 (2001) .

[3] E. Gorlier, J.M. Haudin, J.F. Agassant, J.L., Lepage, G. Perez, D. Darras, N. Billon, The 14th International ESAFORM Conference on Material Forming, Liège, 345-348 (2001).

[4] L. Chevalier, Y. Marco, Int. J. Mech. Mater., 39(6), 596-609, (2006). 
[5] M. Bordival, F.M. Schmidt, Y. Le Maoult, V. Velay, Pol. Eng. Sci., 49(4):783-793 (2009).

[6] B. Cosson, L. Chevalier, J. Yvonnet, Int. Polymer Process, 24(3), 223-233 (2009).

[7]. L. Chevalier, Y. Marco, G. Regnier, Mec. Ind., 2, 229-248 (2001).

[8] F.M.Schmidt, J.F. Agassant, M. Bellet, L. Desoutter, Journal of Non-Newtonian Fluid Mechanics, 64 (1), 19-42 (1996).

[9] G. Barakos, E. Mitsoulis, J. Non-Newt. Fluid Mech., 58, 315-329 (1995).

[10]. B. Debbaut, B. Hocq, and J. M. Marchal, SPEANTEC Tech. Papers, 39, 1870-1872 (1993).

[11] C.P. Buckley, D.C. Jones, Polymer, 36, 3301-3312 (1995).

[12] C.P. Buckley, D.C. Jones, and D.P. Jones, Polymer, 37, 2403 -2414 (1996).

[13] G.H. Menary, C.G. Armstrong, R.J. Crawford, and J.P. McEvoy, Rubber and Composites Processing and Applications, 29, 360-370 (2000).

[14] L. Figiel, C.P. Buckley, International Journal of Non-linear Mechanics, 44, 389-395 (2009).

[15] Y.M. Luo, L. Chevalier, E. Monteiro, The 14th International ESAFORM Conference on Material Forming, Queen's University, Belfast, Irlande du Nord, April 27-29, (2011).

[16] L. Chevalier, Y.M. Luo, E. Monteiro, G. Menary, Mechanics of Material, 52, 103-116, (2012).

[17] G.H. Menary, C.W. Tan, E.M.A. Harkin-Jones, C.G. Armstrong, P.J. Martin, Polymer Engineering \& Science, 52(3), 671-688 (2012).

[18] K. Chung, Journal of Materials Shaping Technology, 7(4), 229-239, (1989).

[19] F.M. Schmidt, J.F. Agassant, and M. Bellet, Polym. Eng. Sci., 38(9), 1399 (1998).

[20] Z.J. Yang, E. Harkin-Jones, G.H. Menary, and C.G. Armstrong, Polym. Eng. Sci., 44, 1379 (2004). 
[21] Z.J. Yang, E. Harkin-Jones, G.H. Menary, and C.G.Armstrong, Journal of Materical Processing Technology 153(154), 20-27, (2004).

[22] F.M. Schmidt, Y. Le Maoult, S. Monteix, Journal of Materials Processing Technology, 143(144), 225-231 (2003)

[23] S. Andrieu, Etude expérimentale et numérique du chauffage infrarouge de plaques thermoplastiques pour le thermoformage, Thèse de Doctorat, ENSMP, (2005).

[24] F. Brezzi, M. Fortin, Mixed and hybrid Finite Element Methods, Springer :New York, (1991).

[25] R.Siegel and J.R.Howell, Thermal Radiation Heat Transfer, 3rd Edition, Hemisphere Publishing Corporation, (1992).

[26] F.M. Schmidt, Y. Le Maoult, S. Monteix, Journal of Materials Processing Technology, 143(144), 225-231 (2003).

[27] B. Cosson, F. Schmidt, Y. Le Maoult, M. Bordival, Int. J. Mater. Form., 4, 1-10, (2011).

[28] J. Sacadura, Initiation aux transferts thermiques. Lavoisier, France. (1973).

[29] M. Bordival, Modélisation et optimisation numérique de l'étape de chauffage infrarouge pour la fabrication de bouteilles en PET par injection-soufflage, Thèse de doctorat, Mines Tech Paris, (2009).

[30] S. W. Churchill, H. S. Chu, International Journal of Heat and Mass Transfer, 18, 1323$1329(1975)$.

[31] Michael F. Modest, Radiative heat transfer, second edition. McGraw-Hill, Inc, (1993). 


\section{List of tables}

Table (1). The numerical value of $G_{0}$

Table (2). The characteristics of the PET

Table (3). The value of thermal properties

Table (4). The values of incident heat flux

Table (5). The characteristics of the PET

Table (6). Errors between the experimental and the results of the model

\section{List of figures}

Figure 1: The hevolution versus the equivalent viscous strain $\bar{\varepsilon}_{v}$ when $m=0.25 .[15,16]$

Figure 2: The 2D rectangular domain with the boundary conditions

Figure 3: (a) The experimental data (+) and the finite elements results of the visco-elastic model (lines) at $90^{\circ} \mathrm{C}$ under different strain rates; (b) The experimental data $(+)$ and the finite elements results of the visco-elastic model (lines) at $8 \mathrm{~s}^{-1}$ under different temperatures.

Figure 4: (a) Experimental heating set-up; (b) IR lamps and PET sheet

Figure 5: Heat capacity $C_{p}$ versus the temperature and the illustration of the $C_{p}$ function

Figure 6: The experimental results (dots) and the numerical results (curves) with optimal thermal properties: (a) $d=11 \mathrm{~cm} ;(b) d=12 \mathrm{~cm} ;$ ( c) $d=13 \mathrm{~cm}$.

Figure 7: The evolution of $C_{p}$ with the parameters identified

Figure 8: (a) Geometrical configuration of the lamps and PET sheet; (b) position of the lamps.

Figure 9: The intensity of the incident radiation calculated by Eq.26 (a) d=11cm; (b) $d=12 \mathrm{~cm} ;(c) d=13 \mathrm{~cm}$. (d): The incident heat flux $\phi_{\lambda 0}$ identified (the points) compared to $\phi_{\lambda 0}$ calculated in the central zone (the line)

Figure 10: The experimental data (the points) and the thermo-mechanical results

Figure 11: (a) The experimental data (the points) and the thermo-mechanical results; (b) The evolution of temperature under different strain rates; (c) The calculated evolution of the viscosity under different strain rates during the biaxial test including the temperature effect. 


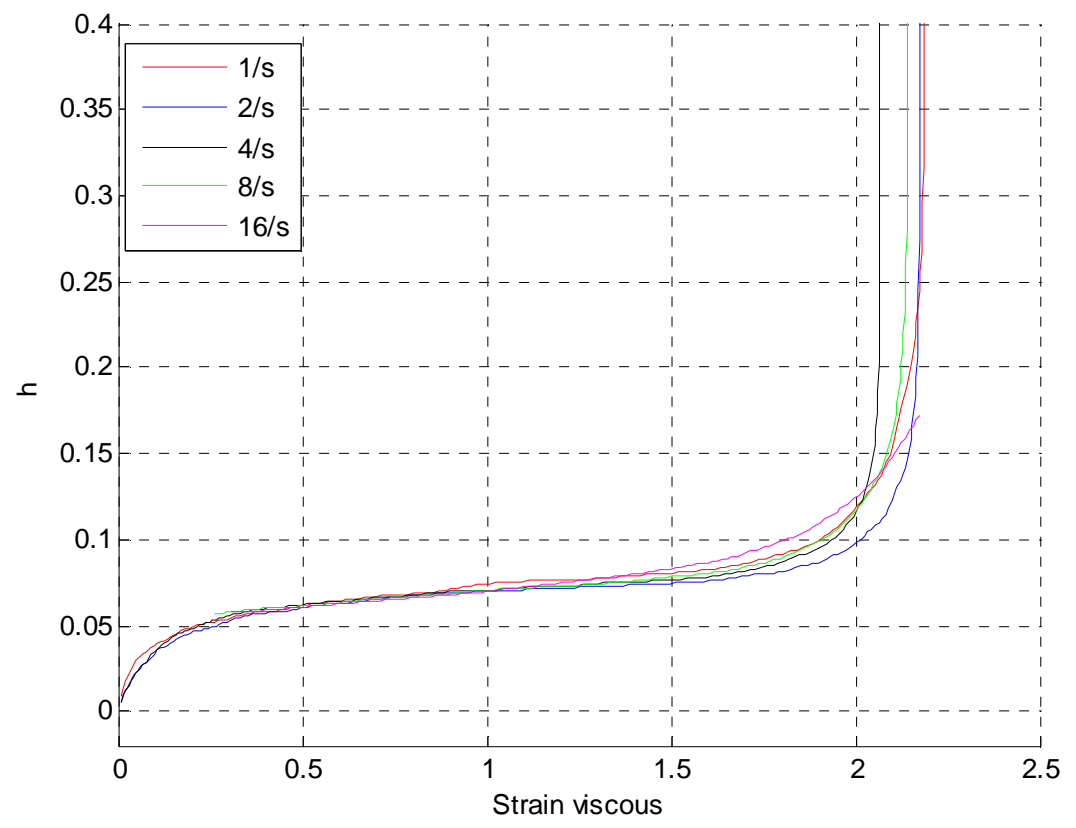

Figure 1: The hevolution versus the equivalent viscous strain $\bar{\varepsilon}_{v} \cdot[15,16]$

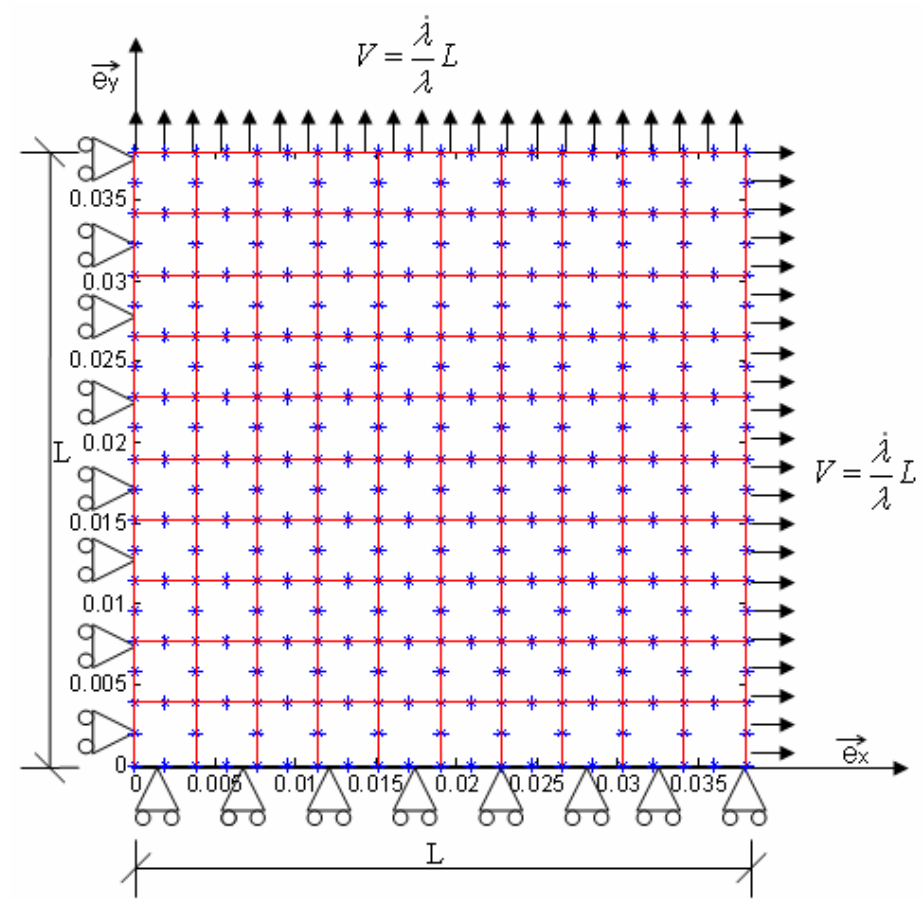

Figure 2: The 2D rectangular domain with the boundary conditions 

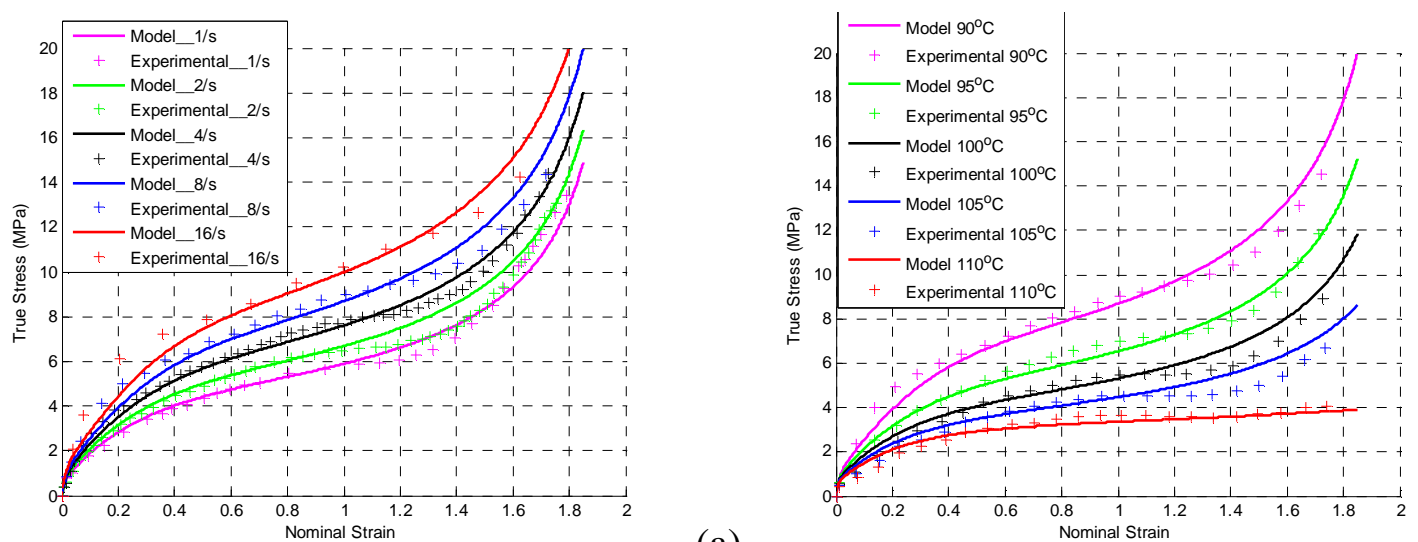

(a)

Figure 3: (a) The experimental data (+) and the finite elements results of the visco-elastic model (lines) at $90^{\circ} \mathrm{C}$ under different strain rates; $(b)$ The experimental data $(+)$ and the finite elements results of the visco-elastic model (lines) at $8 s^{-1}$ under different temperatures.

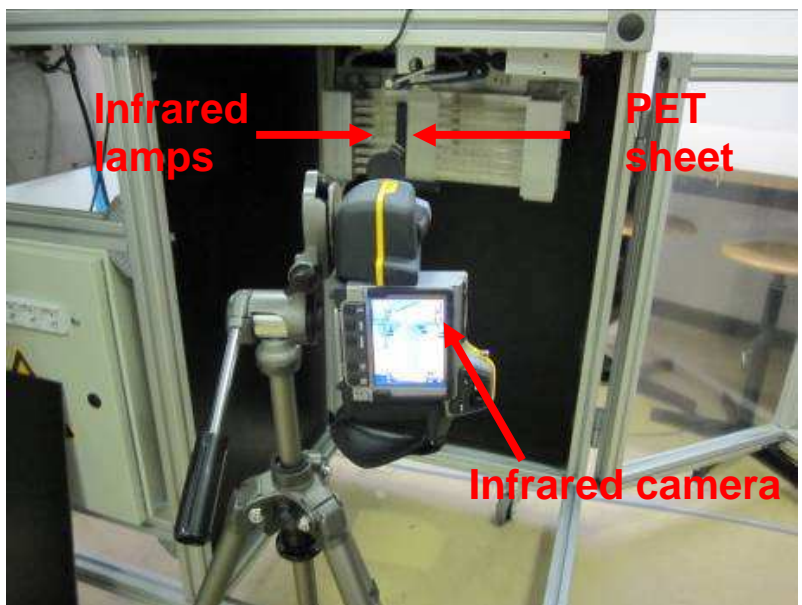

(a)

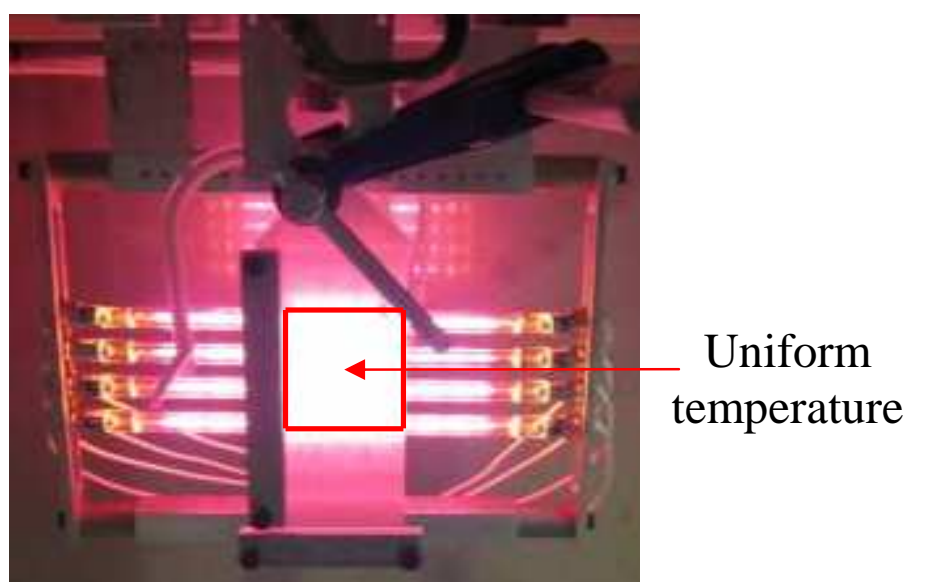

(b)

Figure 4: (a)Experimental heating set-up; (b) IR lamps and PET sheet 


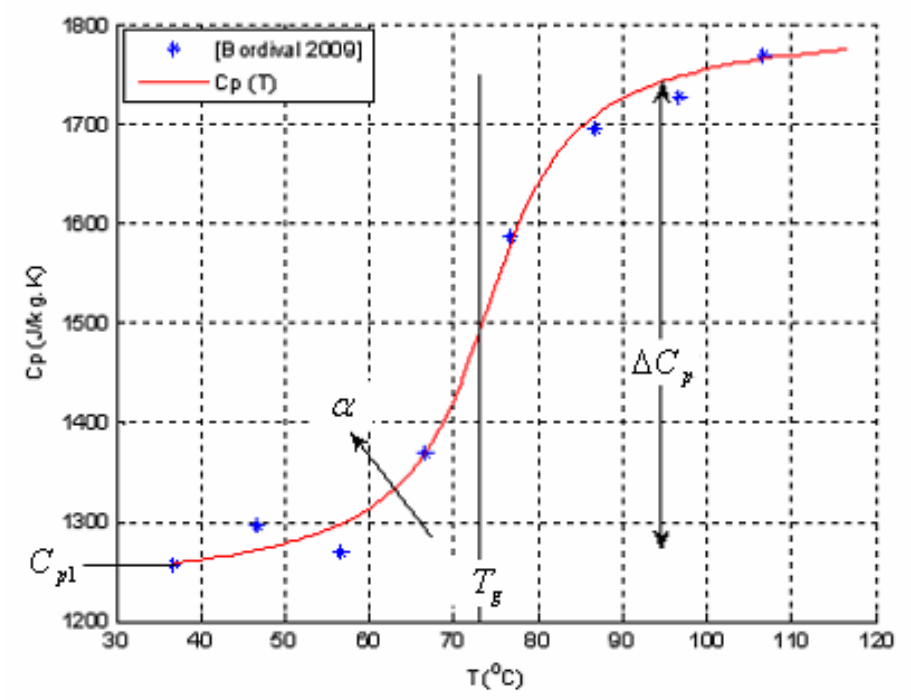

Figure 5: Heat capacity $C_{p}$ versus the temperature and the illustration of the $C_{p}$ function
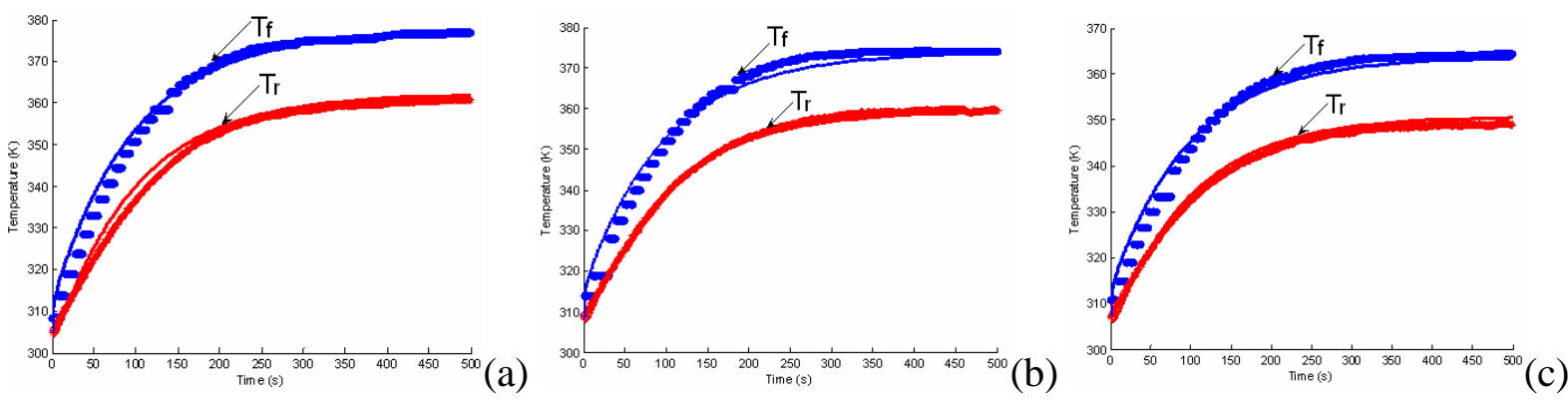

Figure 6: The experimental results (dots) and the numerical results (curves) with optimal thermal properties: (a) $d=11 \mathrm{~cm} ;(b) d=12 \mathrm{~cm} ;(c) d=13 \mathrm{~cm}$.

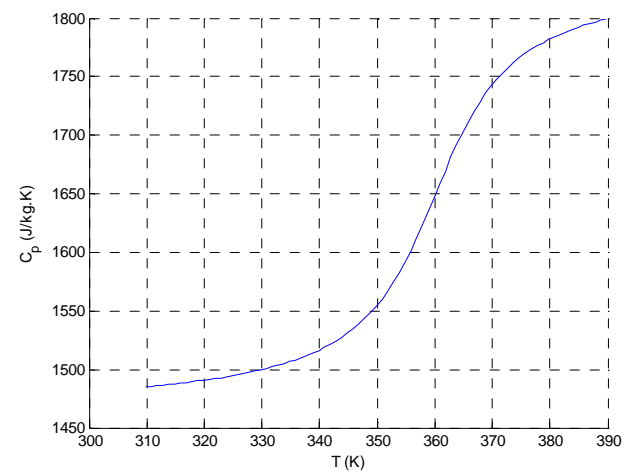

Figure 7: The evolution of $C_{p}$ with the parameters identified 


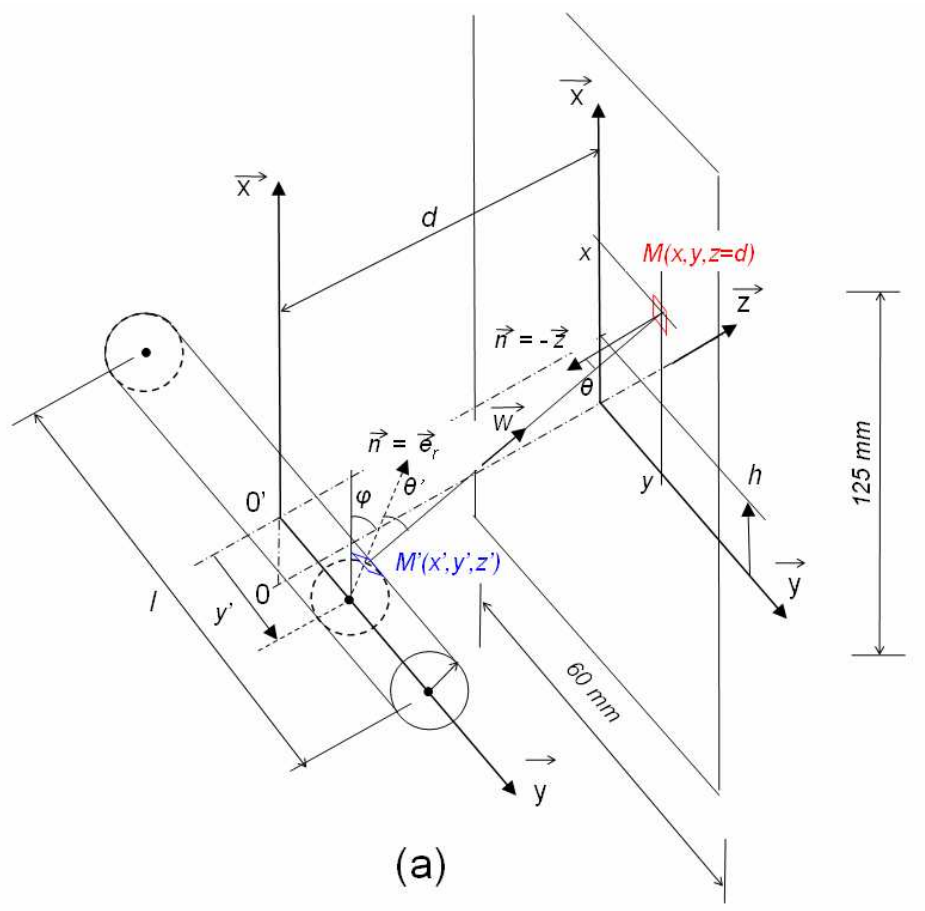

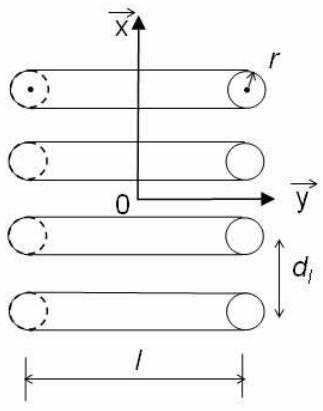

(b)

Figure 8: (a) Geometrical configuration of the lamps and PET sheet; (b) position of the lamps.

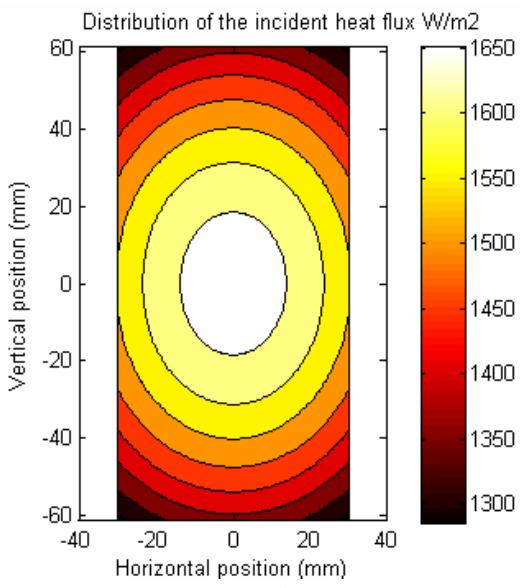

(a)

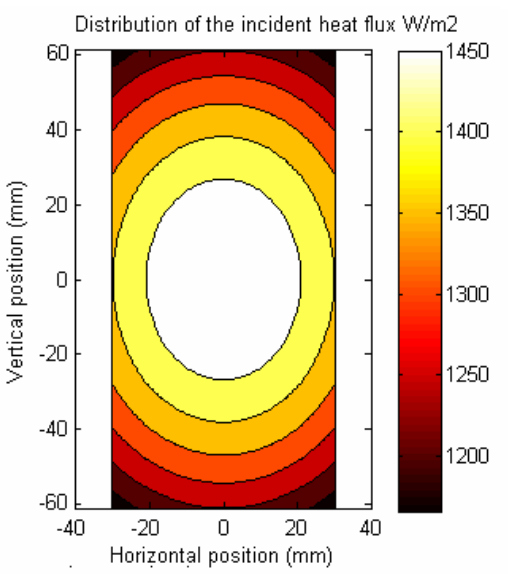

(b)

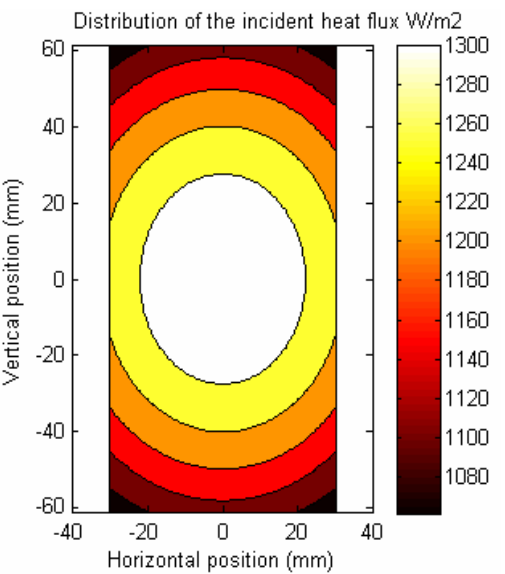

(c)

Figure 9: The intensity of the incident radiation calculated by Eq.26 (a) d=11 cm; (b) $d=12 \mathrm{~cm} ;(\mathrm{c}) \mathrm{d}=13 \mathrm{~cm}$.

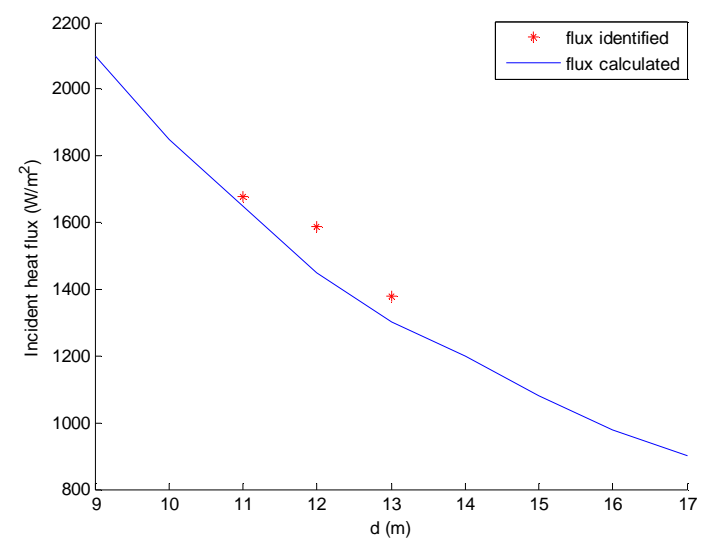


Figure 9d: The incident heat flux $\phi_{\lambda 0}$ identified (the points) compared to $\phi_{\lambda 0}$ calculated in the central zone (the line)

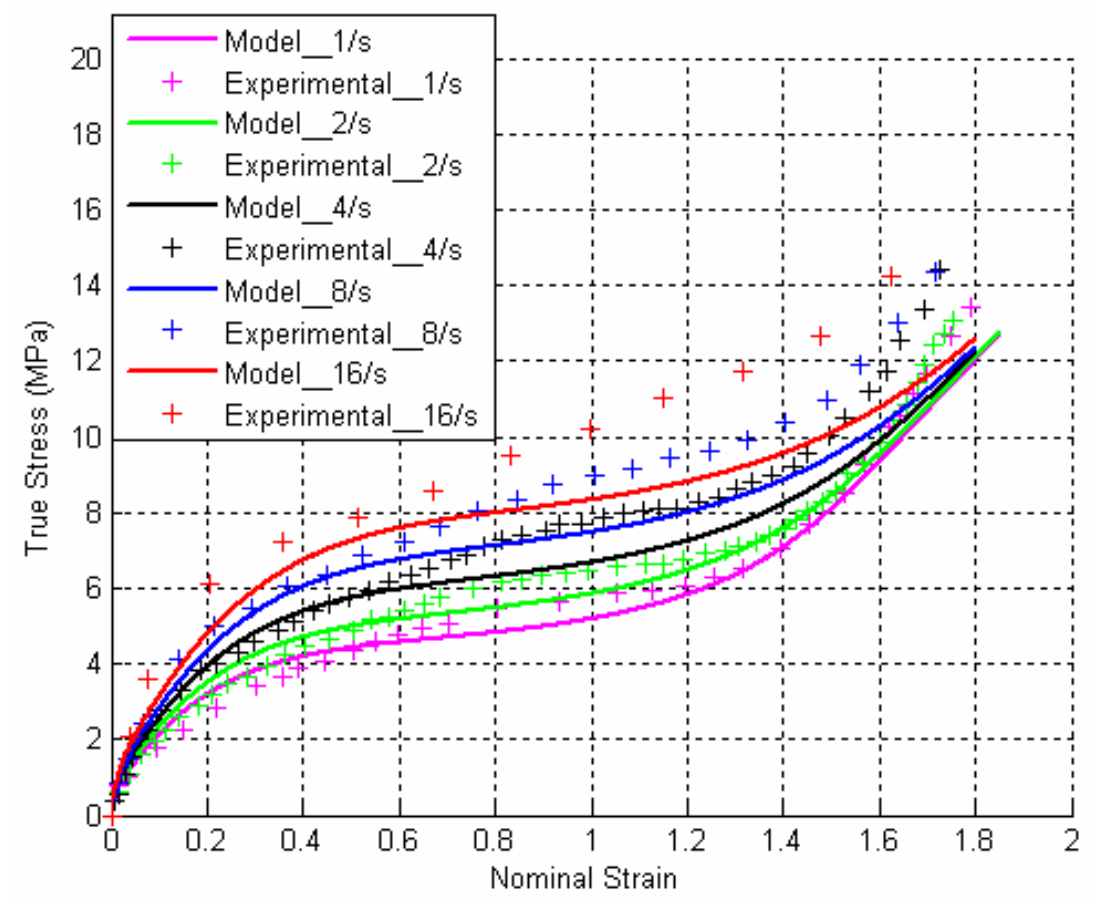

Figure 10: The experimental data (the points) and the thermo-mechanical results

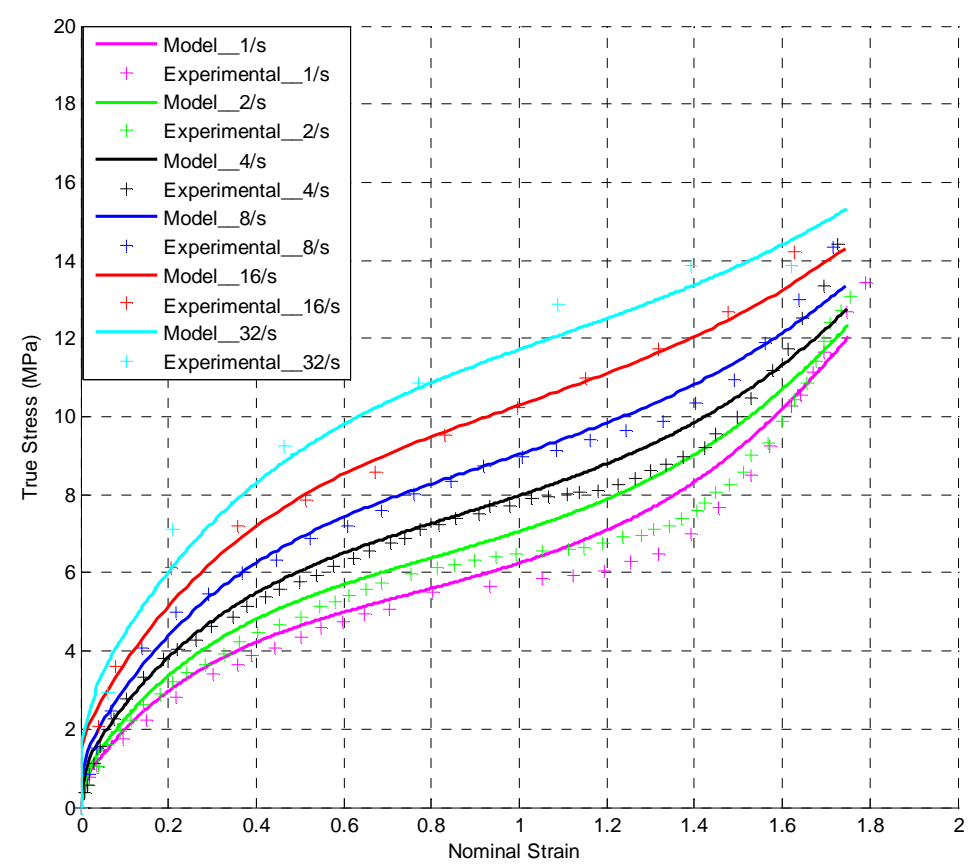




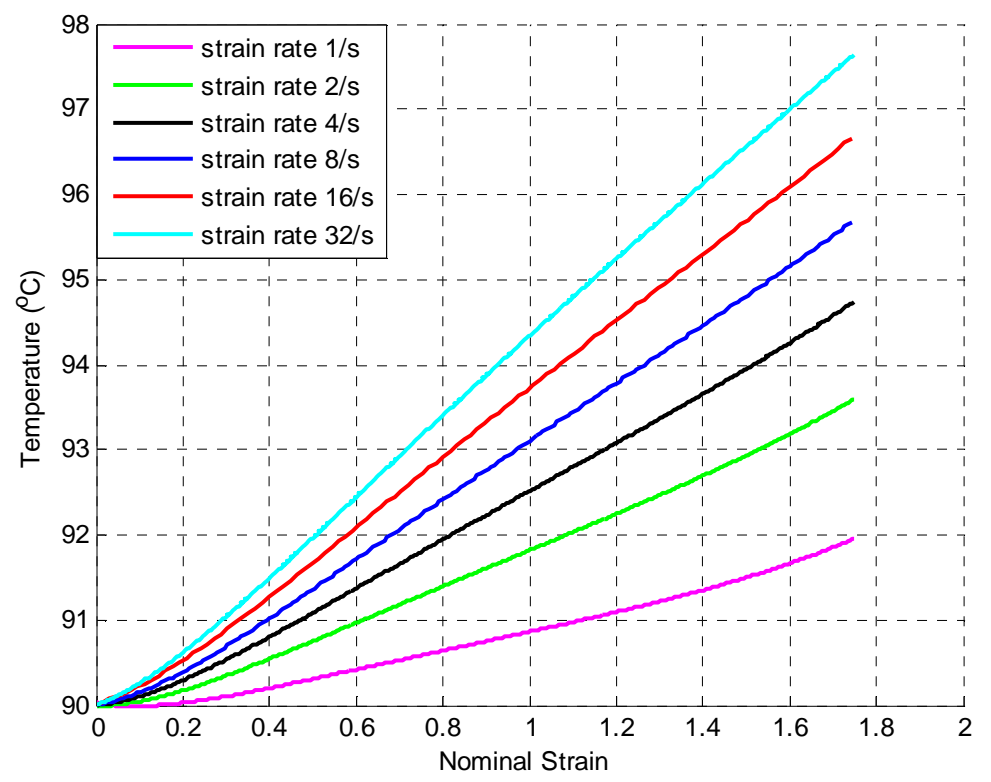

(b)

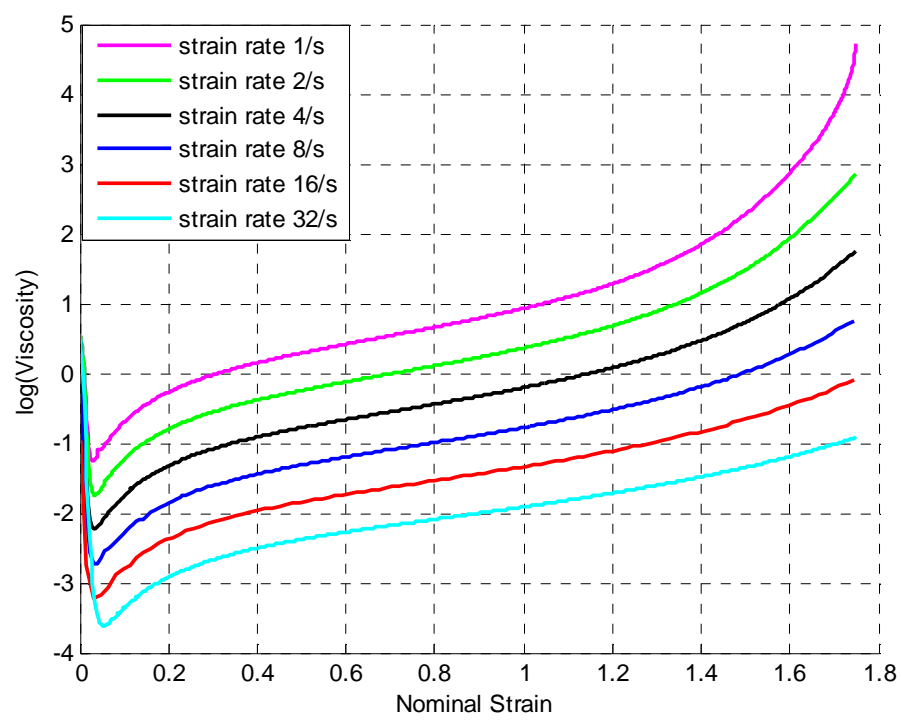

(c)

Figure 11: (a) The experimental data (the points) and the thermo-mechanical results; (b) The evolution of temperature under different strain rates; (c) The calculated evolution of the viscosity under different strain rates during the biaxial test including the temperature effect. 
Table (1). The numerical value of $G_{0}$

\begin{tabular}{l|c|c|c|c|c}
\hline $\begin{array}{l}\text { Strain Rate } \\
(/ \mathrm{s})\end{array}$ & 1 & 2 & 4 & 8 & 16 \\
\hline$G_{0}(\mathrm{MPa})$ & 7.2 & 8.1 & 7.7 & 7.9 & 8.9 \\
\hline $\operatorname{Min} G_{0}(\mathrm{MPa})$ & \multicolumn{5}{|c}{7.2} \\
\hline $\operatorname{Max} G_{0}(\mathrm{MPa})$ & \multicolumn{5}{c}{8.9} \\
\hline
\end{tabular}

Table (2). The characteristics of the PET

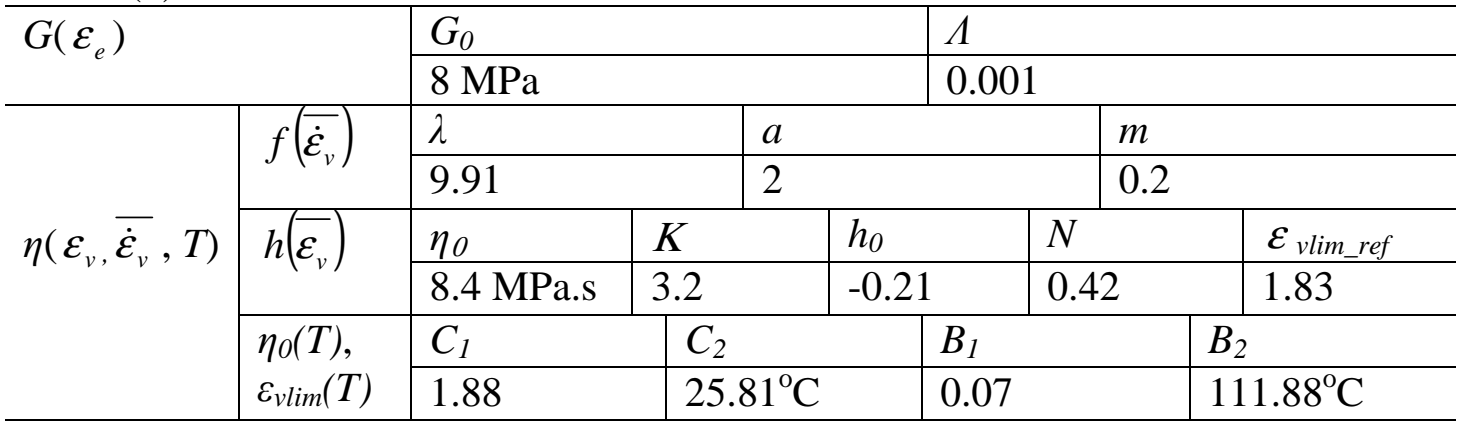

Table (3). The value of thermal properties

\begin{tabular}{l|c|c|c|c|c|c|c|c|c}
\hline \multirow{2}{*}{ Parameter } & $\begin{array}{c}\rho \\
\left(\mathrm{kg} / \mathrm{m}^{3}\right)\end{array}$ & \multicolumn{4}{|c|}{$\begin{array}{c}C_{p} \\
(\mathrm{~J} / \mathrm{kg} \cdot \mathrm{K})\end{array}$} & \multirow{2}{*}{$\begin{array}{c}k \\
(\mathrm{~W} / \mathrm{m} . \mathrm{K})\end{array}$} & \multicolumn{2}{|c|}{$\begin{array}{c}h \\
\left(\mathrm{~W} / \mathrm{m}^{2} . \mathrm{K}\right)\end{array}$} & \multirow{2}{*}{\begin{tabular}{c}
$k_{\lambda}$ \\
\cline { 3 - 5 }$(/ \mathrm{m})$
\end{tabular}} \\
\cline { 3 - 5 } & & $C_{p 1}$ & $T_{g}$ & $\Delta C_{p}$ & $\alpha$ & & $h_{f}$ & $h_{r}$ & \\
\hline Value & 1400 & 120 & 87 & 1650 & 0.1 & 0.07 & 7 & 16 & $3.10^{4}$ \\
\hline
\end{tabular}

Table (4). The values of incident heat flux

\begin{tabular}{l|c|c|c}
\hline$d(\mathrm{~cm})$ & 11 & 12 & 13 \\
\hline$\phi_{\lambda 0}$ & 1650 & 1450 & 1300 \\
calculated $\left(W / m^{2}\right)$ & & & \\
\hline$\phi_{\lambda 0}$ identified & 1680 & 1590 & 1380 \\
$\left(W / m^{2}\right)$ & & & \\
\hline
\end{tabular}

Table (5). The characteristics of the PET

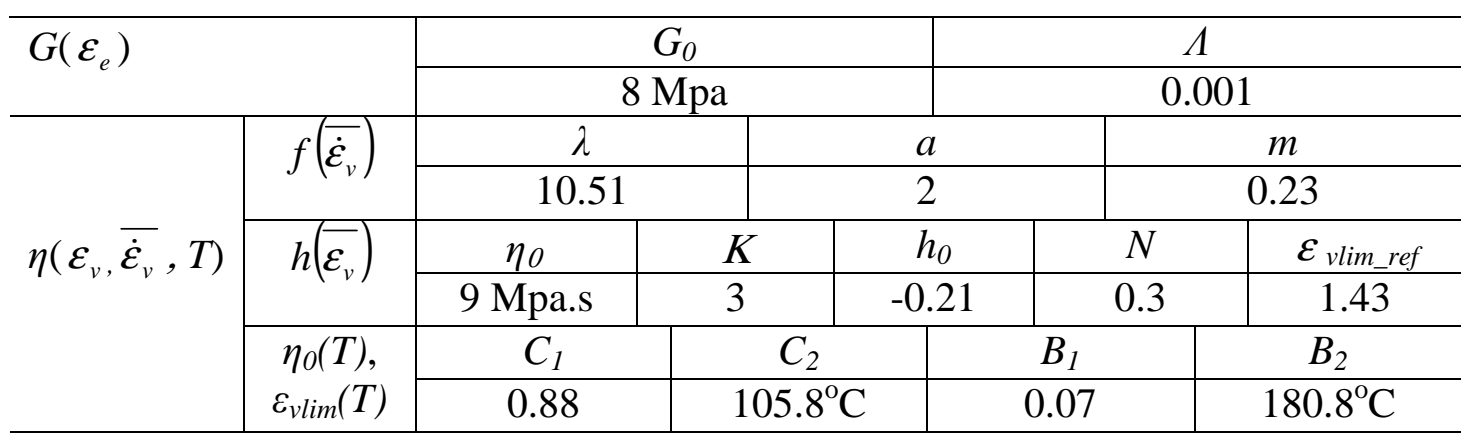


Table (6). Errors between the experimental and the results of the model

\begin{tabular}{c|c}
\hline Strain Rate $(/ \mathrm{s})$ & Relative Error $(\%)$ \\
\hline 1 & 6.72 \\
2 & 8.4 \\
4 & 4.97 \\
8 & 4.45 \\
16 & 8.55 \\
32 & 9.25 \\
\hline
\end{tabular}

